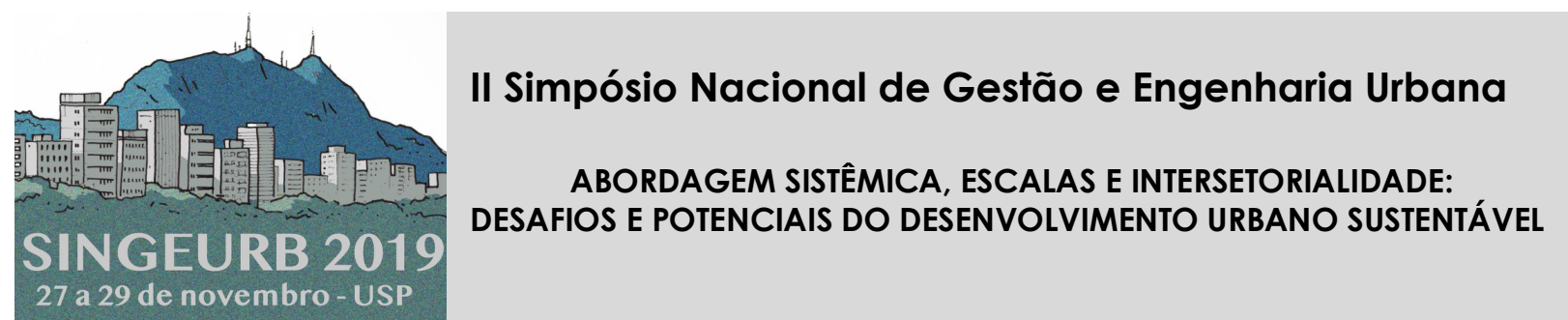

\title{
Os princípios do urbanismo sustentável no desenvolvimento de bairros via IMM: os vazios urbanos dos bairros Jockey e Fátima, em Teresina/PI. ${ }^{1}$
}

\section{The principles of sustainable urbanism in the neighborhoods development via IMM: the Jockey and Fátima's urban voids, in Teresina/PI.}

\author{
Nascimento, Mauro Jonas Cardoso do'; Corrêa, Roberto Machado² \\ 1 Programa de Engenharia Urbana da Escola Politécnica da Universidade \\ Federal do Rio de Janeiro (PEU/POLI/UFRJ), Rua Álvaro Ramos, 319 - Botafogo \\ - RJ, Brasil, mauro.jonas@poli.ufrj.br \\ 2 PEU/POLI/UFRJ, roberto@poli.ufrj.br
}

\begin{abstract}
RESUMO
Nos estudos morfológicos, diversas decisões são tomadas, porém poucas levam em conta a visão sistêmica capaz de compreender e avaliar todos os setores da infraestrutura urbana. Adotar essa visão é entender a cidade como um sistema complexo e, portanto, seria necessário munir-se de técnicas de avaliação e simulação que considerem esse tipo de abordagem. Assim, a IMM - Integrated Modification Methodology, como metodologia sistêmica, baseada na modificação e integração de seus elementos, apresenta-se como uma importante ferramenta capaz de auxiliar na transformação de um contexto urbano existente em outro mais sustentável. Este trabalho faz parte da pesquisa de mestrado sobre os vazios urbanos em dois bairros de Teresina e apresenta a IMM e casos em que ela vem sendo aplicada para avaliar diferentes configurações urbanas. Até o momento, percebeu-se a falta de conectividade entre modais de transporte decorrente dos vazios e usos do solo presentes na área. O objetivo é mostrar o caminho para uma transformação mais sustentável, dentro dessa configuração, após todos os resultados obtidos. Para isso, são usados os princípios de sustentabilidade, no âmbito da Agenda 2030 local de Teresina, como fonte balizadora de uma transformação que traga melhores qualidade de vida e justiça social para as comunidades.
\end{abstract}

Palavras-chave: Urbanismo Sustentável; Morfologia; Integrated Modification Methodology.

\begin{abstract}
In morphological studies, several decisions are taken, but few take into account the systemic vision capable of comprehending and evaluating all specialties of urban infrastructure. Taking this position, cities must be seen as a complex system and, therefore, it would be necessary to

\footnotetext{
${ }^{1}$ NASCIMENTO, Mauro Jonas Cardoso do; CORRÊA, Roberto Machado. Os princípios do urbanismo sustentável no desenvolvimento de bairros via IMM: os vazios urbanos dos bairros Jockey e Fátima, em Teresina/PI. In: II SIMPÓSIO NACIONAL DE GESTÃO E ENGENHARIA URBANA: SINGEURB, 2019, São Paulo. Anais... Porto Alegre: ANTAC, 2019.
} 
have evaluation and simulation techniques that consider these approaches. Hence, the IMM, as a systemic methodology, based on modification and integration of its elements, presents itself as an important tool capable of helping to transform an existing urban context into a more sustainable one. This paper is part of the master's research on the urban voids in two neighborhoods of Teresina and presents the IMM and cases applied by it to evaluate different urban configurations. Heretofore, it has been noticed the lack of connectivity between transportation modals due to the voids and land uses present in the area. The aim is to show the line to a more sustainable transformation, within this configuration, after all the research results. For this, the principles of sustainability are used, within the framework of the Teresina's Local Agenda 2030, as hornbook for a transformation that brings better quality of life and social justice for the neighborhoods.

Keywords: Sustainable Urbanism; Morphology; Integrated Modification Methodology.

\section{INTRODUÇÃO}

Pensar as cidades no contexto atual é não prescindir de novas visões - complexidade e abordagem sistêmica - que levam em conta todas as infraestruturas urbanas, os stakeholders e suas atribuições, bem como os aspectos econômicos, sociais, ambientais, políticos e culturais. São eles que fundamentam os novos princípios de planejamento e gestão urbanos.

O presente artigo busca mostrar como esses novos princípios, inerentes a sustentabilidade, vêm sendo aplicados na transformação da morfologia urbana por meio de um método multiestágio, propondo sua aplicação numa transformação sustentável de bairros, tendo como contexto vazios urbanos.

\section{CONTEXTO}

O recorte espacial deste trabalho está localizado na cidade de Teresina, capital do estado do Piauí, e suas primeiras formas de povoamento ocorreram de forma não planejada, tendo como principais marcos: as construções do Jockey Clube do Piaú - antigo hipódromo - e da Capela e do Centro Social de Nossa Senhora de Fátima (ARAÚJO, 2009).

Nesse contexto, o crescimento urbano de Teresina foi marcado por um acentuado processo de migração das populações com maior poder aquisitivo para essa área - zona leste - em paralelo à construção de grandes conjuntos habitacionais - na zona sul - para a população de baixa renda, incorporando ao espaço intraurbano grandes áreas vazias (LIMA, 2002).

Assim, essas diferentes formas de ocupação do território determinaram duas lógicas de formação dos vazios em Teresina. Embora a formação desses vazios esteja associada aos interesses especulativos, um foi marcado por forte atuação do Estado na sua formação - zona sul - e o outro - zona leste - marcado pela atuação de agentes particulares, embora haja também, forte atuação do Poder Público nesse processo.

\section{VAZIOS URBANOS}

Para análise do contexto urbano deste trabalho, adota-se o conceito de terrenos vagos da Prefeitura Municipal de Teresina - PMT, que leva em conta a ideia de terreno (solo urbano) não edificado, não utilizado ou subutilizado, para fins de aplicação do IPTU progressivo no tempo, da seguinte forma:

- Solo urbano não edificado: aquele que, situado na zona urbanizada, com área igual ou superior a $500 \mathrm{~m}^{2}$ (quinhentos metros quadrados), apresenta índice de aproveitamento igual a zero, conforme Lei Complementar $n^{\circ} 3.562 / 2006$ (TERESINA, 2015);

- Solo urbano não utilizado: aquele edificado, mas, comprovadamente desocupado há mais de dois anos, ressalvado os casos dos imóveis integrantes de massa falida (TERESINA, 2015);

- Solo urbano subutilizado: aquele que, situado na zona urbanizada, com área igual ou superior a $500 \mathrm{~m}^{2}$ (quinhentos metros quadrados), apresenta área construída inferior a 10\% 
(dez por cento) da área do terreno (TERESINA, 2015).

\section{URBANISMO SUSTENTÁVEL - US}

Existe uma ampla bibliografia que trata de princípios, parâmetros ou diretrizes com o objetivo de alcançar o desenvolvimento sustentável. Assim, urbanistas, pesquisadores e decisionmakers que buscam uma requalificação das comunidades devem não só levar em conta esse universo bibliográfico, mas também, as especificidades de cada local.

Adota-se como referencial os princípios de Farr (2013), que toma o bairro como unidade básica do planejamento urbano, sendo mais fácil alcançar a sustentabilidade, quando aquele integrar cinco atributos: definição, compacidade, totalidade, conexão e biofilia.

Segundo Farr (2013) o US tem sua origem em três movimentos de reforma do final do século XX - Smart Growth, New Urbanism e Green Building - e são cinco os parâmetros emergentes para esse urbanismo:

- aumento da sustentabilidade com o aumento da densidade urbana;

- corredores de sustentabilidade;

- bairros sustentáveis;

- Biofilia e

- edificações e infraestrutura de alto desempenho.

\section{CASOS E ESTUDO DE CASO}

Aqui, expõe-se alguns trabalhos já desenvolvidos com as ideias do US que aplicaram a tecnologia Integrated Modification Metodology - IMM, um processo multi-estágio, na análise de contextos urbanos vistos como Sistemas Complexos Adaptáveis - SCA.

A IMM aplica princípios de projetos - DOPs (Design Ordering Principles) como ferramentas de organização estrutural do SCA que estão ligados às ideias do US. Estes DOPs estão associados a indicadores, com o objetivo de comparar o desempenho do SCA antes e depois da transformação (TADI et al, 2017). Ela é composta por quatro fases totalmente integradas:

- Investigação;

- Formulação;

- Modificação;

- Otimização.

Na primeira fase, investiga-se a configuração e as características atuais de um SCA, considerando que a cidade é composta basicamente por quatro Subsistemas Principais - SP: Volumes, Vazios, Funções e Transportes, que interagem de forma dinâmica delineando uma morfologia específica (Ver Figuras 1 e 2). Essa interação cria determinantes chamados de Categorias Chave (CC) ou primeiro nível de sobreposição das CC e ocorre da seguinte forma:

- Volumes + Vazios $\rightarrow$ Porosidade;

- Volumes + Funções $\rightarrow$ Proximidade;

- Vazios + Funções $\rightarrow$ Diversidade;

- Vazios + Transportes $\rightarrow$ Interface;

- Transportes + Volumes $\rightarrow$ Eficiência;

- Transportes + Funções $\rightarrow$ Acessibilidade. 
Figura 1 - Subsistemas Principais

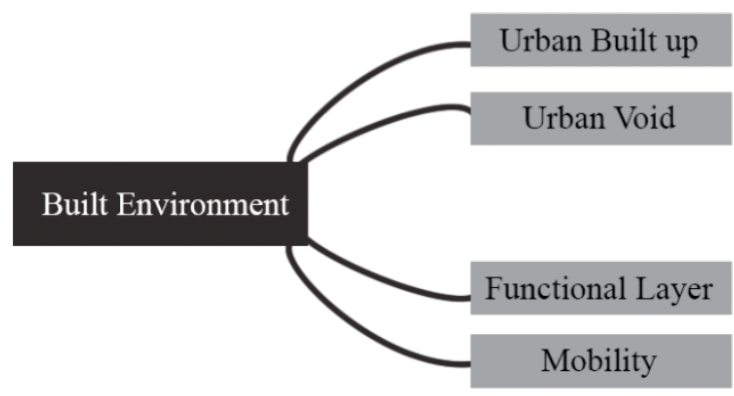

Fonte: Tadi et al (2017)

Figura 2 - Categorias Chaves

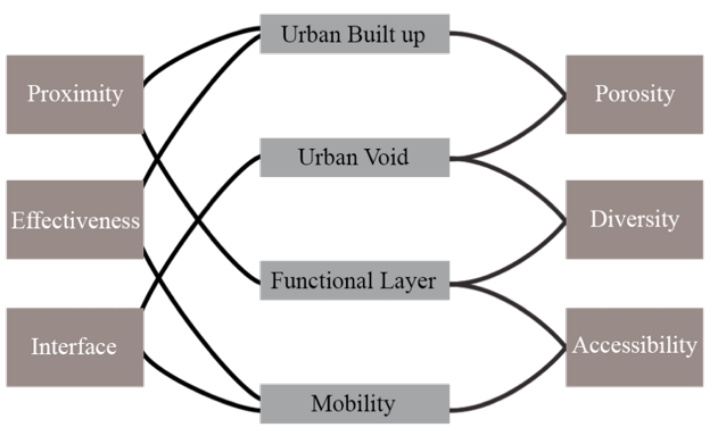

Fonte: Tadi et al (2017)

O segundo nível de sobreposição consiste no cruzamento dos três pares de CC, sendo:

- Porosidade $x$ Proximidade $\rightarrow$ Compacidade;

- Interface x Eficácia $\rightarrow$ Conectividade;

- Diversidade $\times$ Acessibilidade $\rightarrow$ Complexidade.

Os resultados do primeiro e segundo nível de sobreposição das CC habilita o projetista, por meio de um equilíbrio correto, a alcançar uma forma mais sustentável (TADI E VAHABZADEH MANESH, 2013).

Já a fase 2 visa estabelecer uma hipótese, por meio de catalisadores: os SP e CC com defeitos são escolhidos como catalisadores responsáveis pela transformação do processo. A Fase 3 é o ponto de partida de uma reação em cadeia, orientada por uma modificação local em direção à transformação global do SCA (TADI E BOGUNOVICH, 2017).

Na fase 4, o novo CAS será avaliado e comparado com o anterior por meio dos indicadores aplicados na primeira fase (TADI E VAHABZADEH MANESH, 2013).

\subsection{New Lynn}

New Lynn é um subúrbio residencial de baixa densidade situado em Auckland, Nova Zelândia que tem a expansão urbana como forma dominante de crescimento.

Considerando um contexto desafiador para testar o IMM, Tadi e Bogunovich (2017), ao aplicálo em New Lynn, observa melhorias na conectividade por meio da rede de bicicletas ligando dois novos grandes centros de emprego (acessibilidade $x$ eficácia). A densificação proposta para o centro com uma maior distribuição de funções chave (porosidade $x$ proximidade) permitem, também, uma evolução de compacidade. Por fim, uma maior concentração de funções nos dois núcleos (diversidade) somada à ligação entre eles por funções ao longo do novo eixo de transportes (interface) permitem aumento de complexidade e compacidade. 
Por fim, uma maior concentração de funções nos dois núcleos (diversidade) somada à ligação entre eles por funções ao longo do novo eixo de transportes (interface) permitem aumento de complexidade.

\subsection{Porto Maravilha}

O Porto Maravilha é um projeto realizado no Rio de Janeiro destinado à recuperação da infraestrutura urbana, dos transportes, do meio ambiente e dos patrimônios histórico e cultural de uma grande parte da Região Portuária.

Tadi et al (2015) destaca a correlação entre a morfologia urbana e os desempenhos ambientais naquela área, aplicando IMM. Na comparação foi possível visualizar melhoria de $21 \%$ na interface por conta da ligação entre três morros pertencentes a área. Também há melhorias na Porosidade, Proximidade, Acessibilidade e Diversidade bem como uma reação da Eficácia por conta do novo equilíbrio de uso do solo - quebra das superquadras - e a nova rede de transporte

\subsection{Enseada do Suá}

O bairro Enseada do Suá surgiu de um plano de urbanização cujo objetivo foi criar uma nova área de ampliação do comércio e serviço na cidade de Vitória. Era um antigo aterro com área de 1.300,00 m2.

Negreiros (2017) ligando os princípios de projeto com os de Smart Cities, observa melhorias no acesso aos comércios e serviço por meio da caminhada (proximidade)e permeabilidade do tecido urbano por conta de novas ligações de ruas (interface).

\subsection{Estudo de Caso}

Aplicando-se a IMM numa determinada área, com forte presença de vazios, dos bairros Jockey e Fátima será feita uma análise de como tornar aquele SCA um ambiente mais sustentável.

Para tanto, tem-se como referência os princípios ligados a sustentabilidade da Agenda 2030 - importante documento organizado em cinco eixos: sustentabilidade, oportunidades, direitos, criatividade e governo eficiente - que estabelece as diretrizes para 0 desenvolvimento de Teresina até 2030. São eles que embasarão a formulação dos DOPs do estudo de caso.

A seguir, as primeiras observações levantadas, de acordo com dados levantados junto à Secretaria Municipal de Finanças de Teresina - SMF:

- Ao analisar o mapa de Volumes e Vazios (Figuras 3 e 4), chamam a atenção o grande terreno vazio ao norte e os terrenos vazios muito próximos, ao sul cujas áreas totalizam respectivamente $88.596,12 \mathrm{~m}^{2}$ e $54.831,73 \mathrm{~m}^{2}$.

- Já no mapa Transportes (Figura 5), observa-se que a região só é servida de transporte público de ônibus e que a ciclovias não apresentam conexão com esse modal, nem existem bicicletários próximos às paradas. Há uma divisão física entre as regiões norte e sul da área: acredita-se que essa desconexão seja por conta dos vários terrenos vazios ao longo da Avenida Raul Lopes pelo lado leste, haja vista a falta de atividades fortes nessa região, a rede de transporte público limita-se apenas a servir as porções norte e sul.

- Essa grande quantidade de terrenos vazios corresponde a um deserto funcional (Figura 6). A área em volta do maior terreno vazio, ao norte, é a parte mais afetada por esse deserto funcional. Diferentemente, a área em volta dos terrenos vazios, na porção sul, não é tão atingida assim, isso porque estes estão situados num trecho de convergência das avenidas que limitam a área de intervenção. Além disso, a avenida do lado direito que se encontra mais próximo daqueles terrenos - Avenida Ininga - possui diversas atividades ao longo de seu eixo, indo da porção sul até a porção norte. 
Figura 3 - Volumes

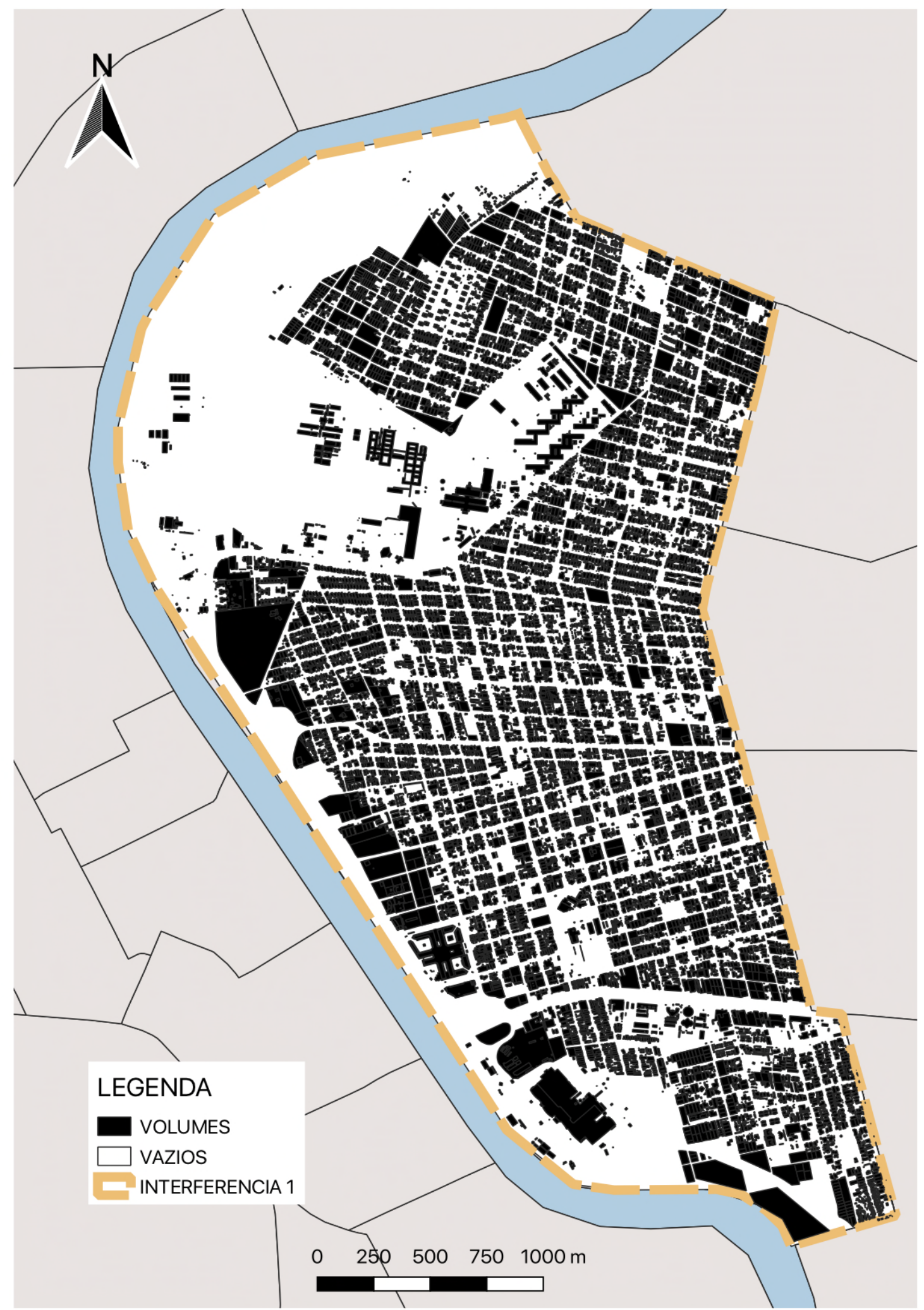

Fonte: O autor (2018) 


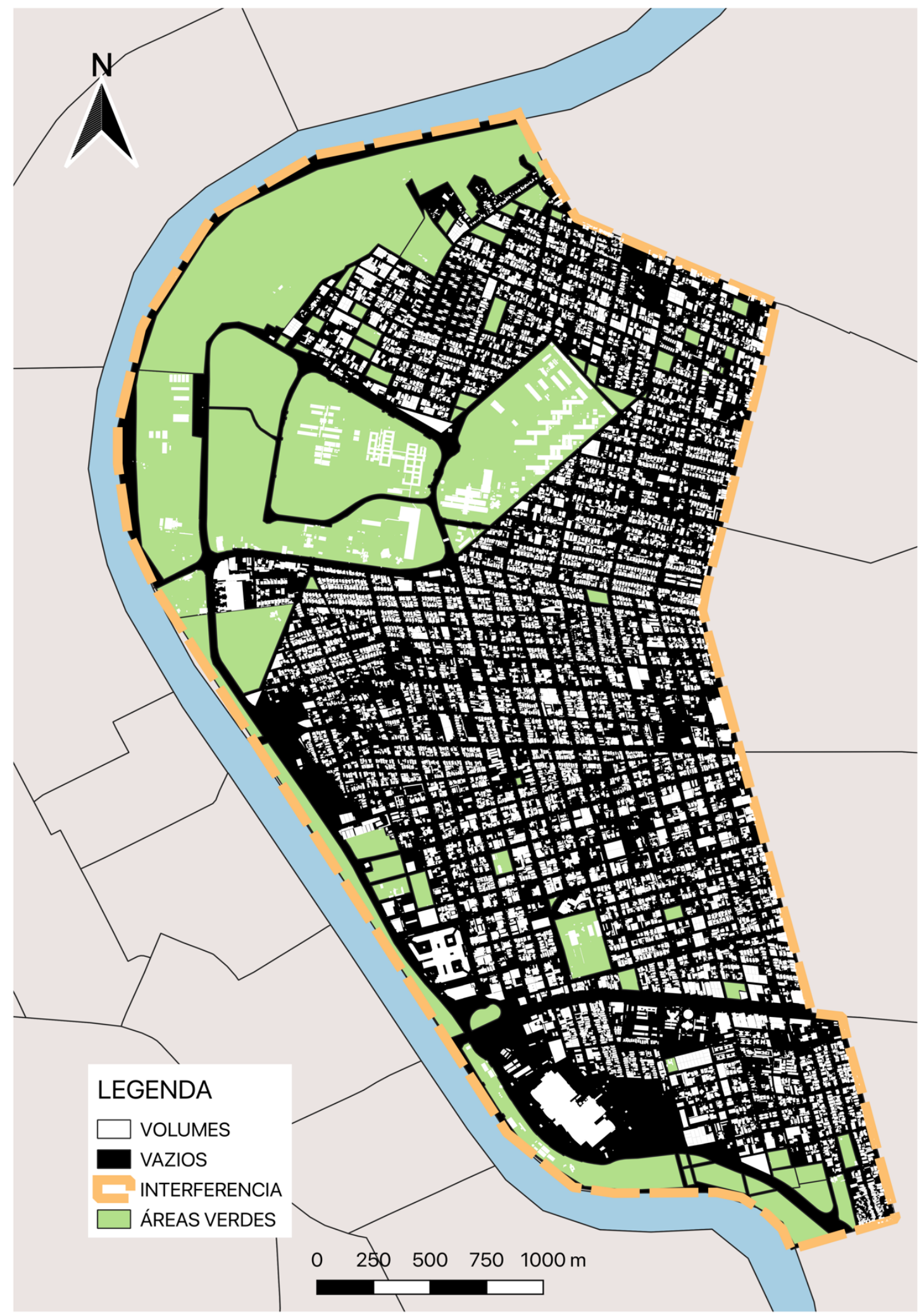

Fonte: O autor (2018) 
Figura 5 - Transportes

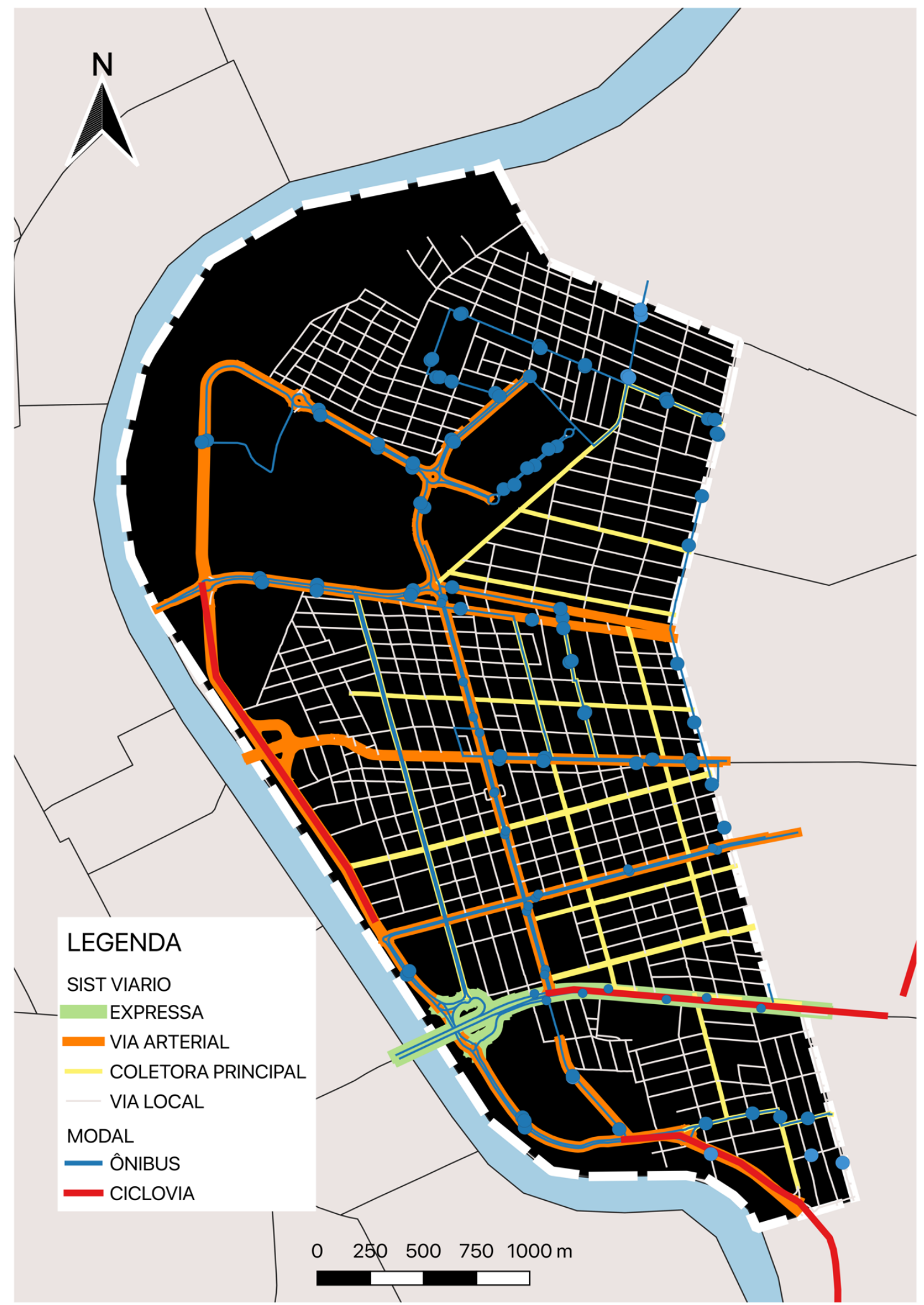

Fonte: O autor (2018) 


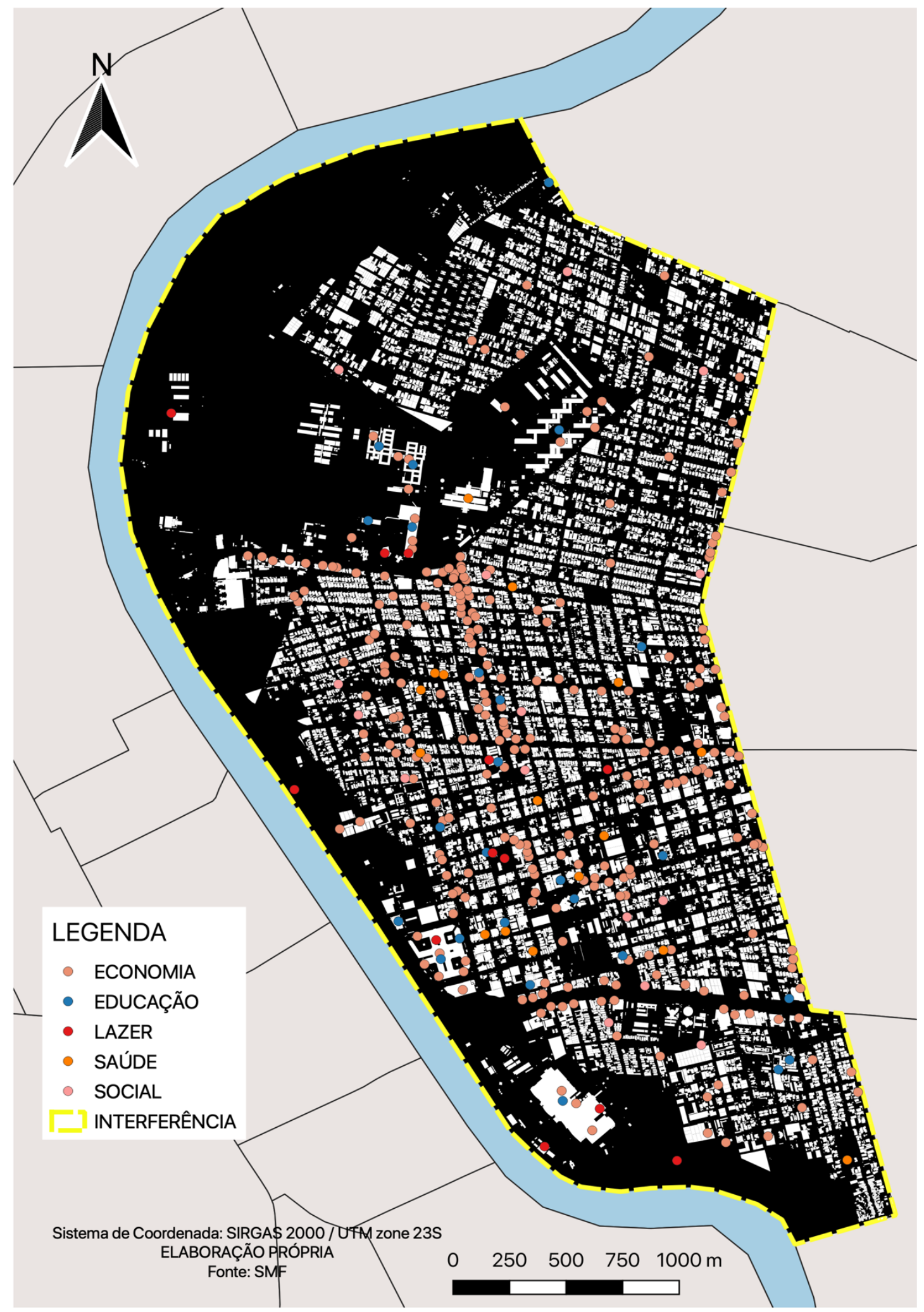

Fonte: O autor (2018) 


\section{CONCLUSÕES}

Conforme estudo do casos citados, a IMM investiga qualquer contexto urbano específico em uma abordagem sistêmica, melhorando o seu desempenho.

Percebe-se que o US trata de princípios que buscam melhor qualidade de vida e justiça social para as comunidades. A IMM é uma tecnologia que, por meio desses princípios, embasa uma modificação urbana, contribuindo através da otimização sistêmica do projeto para enfrentar os desafios do crescimento urbano e de questões ambientais.

Até o momento as primeiras análises são referentes a fase 1 da tecnologia IMM e o objetivo será analisar a modificação do SCA desses bairros, por meio do uso dos princípios ligados a sustentabilidade na elaboração dos DOPs da IMM, como a criação de um ambiente que leve em consideração a escala humana.

Assim, o método auxilia autoridades locais com um desempenho eficaz para bairros ineficientes e de consumo energético espraiado. Quando feita de forma correta, a medição do desempenho pode levar os stakeholders a pensar em questões mais amplas.

Para isso, é preciso entender a cidade como um sistema complexo e usar de metodologias que tenham uma abordagem sistêmica. Isso significa encontrar soluções que tragam benefícios para a sociedade em termos de economia, ambiente, cultura e política.

\section{REFERÊNCIAS}

ARAÚJO, C. C. Trilhas e Estradas: a formação dos bairros Fátima e Jóquei Clube (1960-1980). Dissertação de Mestrado - UFPI. Teresina, 2009.

FARR, D. Urbanismo Sustentável: desenho urbano com a natureza. 1. ed. Porto Alegre: Bookman Editora, 2013. 326p.

LIMA, I. M. M. F. Teresina: urbanização e meio ambiente. Scientia et Spes: Revista do Instituto Camilo Filho, Teresina, PI, v. 1, n. 2, 2002. p. 181-206.

NEGREIROS, P. H. A Cidade Inteligente Bottom-Up: o bairro da Enseada do Suá. Dissertação de Mestrado - UFRJ. Rio de Janeiro, 2017.

TERESINA, Lei Complementar 4.781, de 19 de agosto de 2015. Institui, nos termos dos arts. 50 a 80, da Lei Federal no 10.257, de 10 de julho de 2001 - denominada Estatuto da Cidade -, instrumentos para cumprimento da Função Social da Propriedade no Município de Teresina, e dá outras providências. Diário Oficial do Município, 31 de agosto de 2015, páginas 07 a 09.

TADI, M. \& BOGUNOVICH, D. New Lynn - Auckland IMM Case Study: Low-density urban morphology and energy performance optimisation. Auckland, New Zealand. 2017. Disponível em: http://unitec.ac.nz/epress/. Acesso em: 11 abr. 2019.

TADI, M., BIRAGHI, C., ZADEH, M. H. M., \& BRIOSCHI, L. Urban Porosity. A morphological Key Category for the optimization of the CAS's environmental and energy performance. In: Journal of Engineering Technology. Global Science and Technology Forum, v. 4, n. 3, 2017. p. 138-146.

TADI, M., MANESH, V., MOHAMMAD, M. H., \& ZANIOL, F. Transforming Urban Morphology and Environmental Performances via IMM®. In: Journal of Engineering Technology. Global Science and Technology Forum, v. 3, n. 12, 2015. p. 80-88.

TADI, M., VAHABZADEH MANESH, S. Integrated Modification Methodology (I.M.M): A phasing processor sustainable Urban Design. In: Engineering and Technology. World Academy of Science, 2013. p. 1215-1221. 